\title{
The odd and even intersection properties
}

\author{
Victor Scharaschkin \\ Department of Mathematics \\ University of Queensland \\ St Lucia, Australia, 4072 \\ victors@maths.uq.edu.au
}

Submitted: Dec 16, 2009; Accepted: Sep 7, 2011; Published: Sep 20, 2011

Mathematics Subject Classification: 05A15, 11A15

\begin{abstract}
A non-empty family $\mathcal{S}$ of subsets of a finite set $A$ has the odd (respectively, even) intersection property if there exists non-empty $B \subseteq A$ with $|B \cap S|$ odd (respectively, even) for each $S \in \mathcal{S}$. In characterizing sets of integers that are quadratic nonresidues modulo infinitely many primes, Wright asked for the number of such $\mathcal{S}$, as a function of $|A|$. We give explicit formulae.
\end{abstract}

\section{Introduction}

Let $A$ be a finite non-empty set, let $\mathcal{P}(A)$ denote its powerset, and let $\mathcal{S} \subseteq \mathcal{P}(A)$ be a non-empty collection of subsets of $A$. (We allow $\emptyset \in \mathcal{S}$, but not $\mathcal{S}=\emptyset$.) We say that $\mathcal{S}$ has the even intersection property (EIP) with respect to $A$ if there exists a non-empty set $B \subseteq A$ such that $|B \cap S|$ is even for each $S \in \mathcal{S}$. (The set $B$ is required to be non-empty to avoid triviality.) Similarly, $\mathcal{S} \subseteq \mathcal{P}(A)$ has the odd intersection property (OIP) with respect to $A$ if there exists a set $B \subseteq A$ (necessarily non-empty) with $|B \cap S|$ odd for each $S \in \mathcal{S}$. If $|A|=n$, let $d(n)$, respectively $e(n)$, be the number of $\mathcal{S} \subseteq \mathcal{P}(A)$ with the oIP, respectively the EIP. We shall obtain formulae for $d(n)$ and $e(n)$.

It is generally enough to consider these properties when $A=\bigcup \mathcal{S}$. Indeed, in the odd case, if $\mathcal{S}$ has the OIP with respect to some set $A$ (with appropriate $B \subseteq A$, say) then it has the OIP with respect to $\bigcup \mathcal{S}$, since $B^{\prime}=B \cap \bigcup \mathcal{S}$ still has odd intersection with each $S \in \mathcal{S}$ (in particular, $B^{\prime}$ is not empty). Thus in the odd case we may take $A=\bigcup \mathcal{S}$ without loss of generality, and may simply speak of $\mathcal{S}$ having the OIP (without specifying A).

This observation does not hold in the even case, since $(B \cap \bigcup \mathcal{S})$ may be empty. However if $A \neq \bigcup \mathcal{S}$ then $\mathcal{S}$ has the EIP trivially, since if $x \in A \backslash \bigcup \mathcal{S}$ then $B=\{x\}$ has empty intersection with each $S \in \mathcal{S}$. 
The OIP was introduced by Wright [9], [10] in the following context. If $T \subseteq \mathbb{N}$ is finite and non-empty, there are infinitely many primes $p$ such that every element of $T$ is a quadratic residue mod $p[9$, Theorem 2.3]. Consider the corresponding statement for quadratic nonresidues:

(*) Every element in $T$ is a quadratic nonresidue $\bmod p$, for infinitely many primes $p$.

Wright [9, Lemma 2.5] gave a combinatorial characterization of the sets $T$ satisfying $(*)$. Namely, for each $t$ in $T$ let $S_{t}$ be the set of primes dividing the square-free part of $t$, and let $\mathcal{S}_{T}=\left\{S_{t} \mid t \in T\right\}$. Then (*) holds for $T$ if and only if $\mathcal{S}_{T}$ has the OIP. Recently Hu [6] generalized some of these results to $d$ th powers in the ring $\mathbb{F}_{q}[t]$.

The potential $\mathcal{S}$ with the OIP or EIP are drawn from $\mathcal{P}(\mathcal{P}(A))$, so there are $2^{2^{n}}$ sets to consider and exhaustive searching rapidly becomes impossible. Wright [11] found $d(n)$ for $n \leq 3$, and asked for a general formula.

To state our result, recall [4] that the number of $d$-dimensional subspaces of an $m$ dimensional $\mathbb{F}_{q}$-vector space is given by the q-binomial coefficient

$$
\left(\begin{array}{l}
m \\
d
\end{array}\right)_{q}=\prod_{j=1}^{d} \frac{q^{m-j+1}-1}{q^{j}-1}, \quad 0 \leq d \leq m .
$$

(This expression is always an integer.) We show the following.

Theorem 1.1.

$$
\begin{aligned}
& d(n)=\sum_{i=0}^{n-1}\left[(-1)^{n-i-1}\left(2^{2^{i}}-1\right)\left(\begin{array}{c}
n \\
i
\end{array}\right)_{2} \prod_{j=1}^{n-i}\left(2^{j}-1\right)\right], \\
& e(n)=1+2 \sum_{i=0}^{n-1}\left[(-1)^{n-i-1}\left(2^{2^{i}-1}-1\right)\left(\begin{array}{c}
n \\
i
\end{array}\right)_{2} 2^{\left(\begin{array}{c}
n-i \\
2
\end{array}\right)}\right] .
\end{aligned}
$$

The exponent $\left(\begin{array}{c}n-i \\ 2\end{array}\right)$ in (3) is a regular (not $q$ ) binomial coefficient. The sum in (3) can be interpreted as the number of $\mathcal{S}$ with the EIP with $\emptyset \notin \mathcal{S}$, since except for $\mathcal{S}=\{\emptyset\}, \mathcal{S}$ has the EIP if and only if $\mathcal{S} \cup\{\emptyset\}$ does.

The symmetry between $(2)$ and $(3)$ becomes more apparent on writing $2^{\left(\begin{array}{c}n-i \\ 2\end{array}\right)}=$ $\prod_{j=1}^{n-i-1} 2^{j}$. If we let $\delta=1$ in the EIP case and $\delta=0$ in the OIP case we obtain

$$
d(n), \quad e(n)=\delta+\sum_{i=0}^{n-1}\left[(-1)^{n-i-1}\left(2^{2^{i}}-1-\delta\right)\left(\begin{array}{c}
n \\
i
\end{array}\right)_{2} \prod_{j=1}^{n-i-\delta}\left(2^{j}-1+\delta\right)\right] .
$$

To prove Theorem 1.1 we identify $\mathcal{P}(A)$ with the vector space $V=\mathbb{F}_{2}^{n}$. Equation (2) is then proved in $\S 2$ using linear algebra to establish a recurrence relation satisfied by $d(n)$. Equation (3) is derived in $\S 3$ by a simple counting argument. Except for some notation the two halves of the proof are independent. 
The quantities $d(n)$ and $e(n)$ grow roughly as $2^{\left(2^{n-1}+n\right)}$. The first few values are:

\begin{tabular}{|c|c|c|c|c|c|c|}
\hline$n$ & 1 & 2 & 3 & 4 & 5 & 6 \\
\hline$d(n)$ & 1 & 6 & 63 & 2880 & 1942305 & 270460574370 \\
\hline$e(n)$ & 1 & 7 & 71 & 3071 & 1966207 & 270499994623 \\
\hline
\end{tabular}

and $e(10)>d(10)>10^{150}$.

\section{The Odd Intersection Property}

In this section we prove that $d(n)$ satisfies the following recurrence relation, for $n \geq 2$ :

$$
d(n)=\left(2^{n}-1\right)\left(2^{2^{n-1}}-1-d(n-1)\right) .
$$

The formula (2) for $d(n)$ in Theorem 1.1 follows by solving equation (5), with initial condition $d(1)=1$. The general solution of a first order linear recurrence relation may be found in $[3, \S 1.2]$ or $[7, \S 2.2]$.

In what follows, the disjoint union of sets $S_{1}, \ldots, S_{n}$ is denoted by $\bigsqcup_{i=1}^{n} S_{i}$. To avoid repeating wordy counting arguments, we formalize a trivial observation. If $X$ is a set, $\mathcal{T} \subseteq \mathcal{P}(X)$ and $Q$ is a boolean valued function (predicate) on $\mathcal{P}(X)$, then let $\mathcal{T}^{Q}=\{S \in$ $\mathcal{T} \mid Q(S)$ holds $\}$.

Lemma 2.1. Let $X$ be a non-empty finite set, let $\mathcal{X}=\left\{X_{1}, \ldots, X_{M}\right\} \subseteq \mathcal{P}(X)$, and suppose $\left|\mathcal{P}\left(X_{j}\right)^{Q}\right|=N$ is independent of $j$. For $i \geq 0$ define "level sets"

$$
Z_{i}=\left\{S \in \mathcal{P}(X)^{Q} \mid S \subseteq X_{j} \text { for exactly } i \text { of the } X_{j}\right\}
$$

Then

$$
\mid\left\{S \mid S \subseteq X_{j} \text { for some } j \text {, and } Q(S) \text { holds }\right\}\left|=M N-\sum_{i \geq 2}(i-1) \cdot\right| z_{i} \mid
$$

Proof Clearly $\mid\left\{S \mid S \subseteq X_{j}\right.$ for some $j$, and $Q(S)$ holds $\} \mid$ is just $\left|\bigcup_{X_{j} \in X} \mathcal{P}\left(X_{j}\right)^{Q}\right|=$ $\sum_{i \geq 1}\left|z_{i}\right|$, while $M N=\left|\bigsqcup_{X_{j} \in X} \mathcal{P}\left(X_{j}\right)^{Q}\right|=\sum_{i \geq 1} i\left|z_{i}\right|$.

Suppose $V$ is a finite dimensional $\mathbb{F}_{2}$-vector space and $S$ is a subset of $V$. The subspace of $V$ generated by $S$ is denoted by $\langle S\rangle$. If $v \in V$, then the set $\{v+s \mid s \in S\}$ is denoted by $v+S$. A codimension one subspace ${ }^{1}$ of $V$ is called a maximal subspace. The complement $V \backslash W$ of a maximal subspace $W$ of $V$ is called a $V$-block. A non-empty subset of a $V$-block is called a $V$-subblock.

We define three families of subsets of $V$. Let $\mathcal{M}(V)$ be the collection of all maximal subspaces of $V, \mathcal{B}(V)$ the collection of all $V$-blocks, and $\mathcal{C}(V)$ the collection of all $V$ subblocks:

$$
\mathcal{C}(V)=\bigcup_{B \in \mathcal{B}(V)} \mathcal{P}(B) \backslash\{\emptyset\}
$$

\footnotetext{
${ }^{1}$ The zero space has no maximal subspaces.
} 
In Corollary 2.4 we show that $\mathcal{B}(V)$ forms a symmetric block design, justifying the terminology.

The motivation for introducing these sets is that $|\mathcal{C}(V)|=d(n)$, where $n=\operatorname{dim} V$ (Lemma 2.5). To obtain the recurrence relation (5) we therefore need to consider $V$-blocks, and also $W$-blocks for $W \in \mathcal{M}(V)$ (sets of the form $W \backslash X$ where $X$ has codimension one in $W$ ). We have the following simple properties.

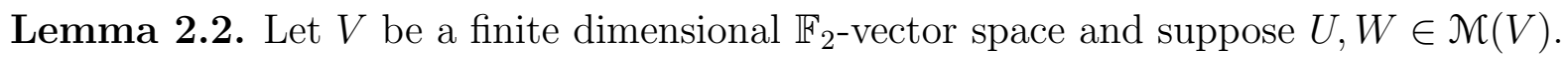

(a) If $U \neq W$ then $U \cap W \in \mathcal{M}(W)$.

(b) We have $\mathrm{e}(W) \subseteq \mathrm{e}(V)$.

(c) Suppose $S$ is a $W$ or $V$-subblock. Then $S \subseteq U$ if and only if $S$ is a $U$-subblock.

\section{Proof}

(a) $U+W$ is all of $V$, so $U /(U \cap W) \simeq(U+W) / W=V / W$ is 1-dimensional.

(b) Suppose $S \in \mathcal{C}(W)$. Say $\emptyset \neq S \subseteq B \subseteq W$ with $W \backslash B \in \mathcal{M}(W)$. Let $x \in V \backslash W$ and $X=\langle x\rangle \oplus(W \backslash B)$. Then $X \in \mathcal{M}(V)$ and $\emptyset \neq S \subseteq V \backslash X$, so $S \in \mathcal{C}(V)$.

(c) By (b) we may assume $S \in \mathcal{C}(V)$. Say $S \subseteq V \backslash X$ for some $X \in \mathcal{M}(V)$. Assume $S \subseteq U$. Then $U \cap X \in \mathcal{M}(U)$ by (a), and $S \subseteq U \backslash(U \cap X)$, so $S \in \mathcal{C}(U)$. The other implication is trivial.

From now on, assume that the set $A$ is fixed, with $|A|=n \geq 1$ and let $V=\mathbb{F}_{2}^{n}$, viewed as a $n$-dimensional $\mathbb{F}_{2}$-vector space. Fix the standard basis $\left\{e_{i}\right\}$ of $V$, where $e_{i}=(0,0, \ldots, 1, \ldots, 0)$ has 1 in the $i$ th coordinate and zeros elsewhere. If $x=\sum x_{i} e_{i}$ and

$y=\sum y_{i} e_{i}$ define $x \cdot y=\sum x_{i} y_{i}$, viewed as an element in $\mathbb{F}_{2}$. (Note that the definition of $x \cdot y$ depends on the basis $\left\{e_{i}\right\}$ chosen.) If $S \subseteq V$ is non-empty, let

$$
\begin{aligned}
& S^{\circ}=\{v \in V \mid s \cdot v=0 \text { for all } s \in S\}, \\
& S^{\prime}=\{v \in V \mid s \cdot v=1 \text { for all } s \in S\},
\end{aligned}
$$

and write $x^{\circ}$ for $\{x\}^{\circ}$ and $x^{\prime}$ for $\{x\}^{\prime}$.

\section{Lemma 2.3.}

(a) The maps $x \mapsto x^{\prime}, x \mapsto x^{\circ}$ give bijections $V \backslash\{0\} \rightarrow \mathcal{B}(V)$ and $V \backslash\{0\} \rightarrow \mathcal{M}(V)$ respectively.

(b) There are $2^{n}-1 V$-blocks each with cardinality $2^{n-1}$, and the same is true for maximal subspaces.

(c) Assume $S \in \mathcal{C}(V)$ or $S \in \mathcal{C}(W)$ for some $W \in \mathcal{M}(V)$, and let $k=n-\operatorname{dim}\langle S\rangle$. Then there are exactly $2^{k} V$-blocks containing $S$, and exactly $2^{k}-1$ maximal subspaces $U$ of $V$ containing $S$. Moreover, in each such $U$ we have $S \in \mathrm{C}(U)$. 
Proof Let $S=\left\{s_{1}, \ldots, s_{m}\right\}$, let $s_{i}=\sum a_{i, j} e_{j}$, and form the $m \times n$ matrix $A=\left(a_{i, j}\right)$ of rank $n-k$. Then $S^{\circ}=\operatorname{ker} A$, so $\operatorname{dim} S^{\circ}=k$. In particular, since $U \subseteq\left(U^{\circ}\right)^{\circ}$ holds for any subspace $U$, taking $S=U$ we have $\operatorname{dim} U=\operatorname{dim}\left(U^{\circ}\right)^{\circ}$, so $U=\left(U^{\circ}\right)^{\circ}$. Thus the maps $\{0, x\} \rightarrow x^{\circ}$ (for $\left.x \neq 0\right)$ and $W \rightarrow W^{\circ}$ give mutually inverse maps between the collection of all 1-dimensional and all maximal subspaces, proving (a) and (b).

Since $\mathrm{e}(W) \subseteq \mathcal{e}(V)$, in (c) we may assume $S$ is a $V$-subblock. By (a), $S^{\prime}=\{x \mid$ $\left.S \subseteq x^{\prime}\right\}$ is (in bijection with) the set of $V$-blocks containing $S$, and hence is non-empty. Furthermore, $S^{\prime}$ is the set of solutions of $A x=\underline{1}$ (the vector of all 1's), so $\left|S^{\prime}\right|=\left|S^{\circ}\right|=2^{k}$.

Finally, let $U \in \mathcal{M}(V)$. By Lemma 2.2(c) $S \subseteq U$ if and only if $S \in \mathcal{C}(U)$. So the number of $U \in \mathcal{M}(V)$ with $S \in \mathcal{C}(U)$ is just the number of maximal subspaces of $V$ containing $S$. Such subspaces are in bijection with the maximal subspaces of $V /\langle S\rangle$, a $k$-dimensional space, containing $2^{k}-1$ maximal subspaces by (b).

Note that (c) above implies that if we know that $S$ is contained in exactly $2^{k} V$-blocks or $2^{k}-1$ maximal subspaces, we can deduce that $\operatorname{dim}\langle S\rangle=k$. We observe in passing that $S \subseteq T^{\circ}$ if and only if $T \subseteq S^{\circ}$, so the $(-)^{\circ}$-operation forms a Galois connection (with itself) $[8$, Ch. 6].

The next result is not needed in our proof, but motivates the terminology. See [5, Ch. 14] or [2, Ch. 1.5] for definitions.

Corollary 2.4. For $n \geq 2$, the set $\mathcal{B}(V)$ forms a symmetric block design on the set $V \backslash\{0\}$, with $(v, k, \lambda)=\left(2^{n}-1,2^{n-1}, 2^{n-2}\right)$.

Proof This follows on putting $S=\{x\}$ and $S=\{x, y\}$ with $x \neq y, x, y \neq 0$ in Lemma $2.3(\mathrm{c})$, since $S^{\prime}$ is clearly non-empty in each case.

We apply these results to the OIP. We identify $\mathcal{P}(A)$ with $V$ by mapping a subset $T \subseteq A=\left\{a_{1}, \ldots, a_{n}\right\}$ to $v_{T}=\sum_{i=1}^{n} \chi_{T}\left(a_{i}\right) e_{i}$, where $\chi_{T}: A \rightarrow \mathbb{F}_{2}$ is the characteristic function of $T$. A collection $\mathcal{S}$ of subsets of $A$ corresponds to a subset $S \subseteq V$. Those collections $\mathcal{S}$ with the OIP correspond to $V$-subblocks:

Lemma 2.5. Under the identification $\mathcal{P}(A) \simeq V$ a collection of subsets of $A$ has the OIP if and only if it corresponds to an element of $\mathcal{C}(V)$. Thus

$$
d(n)=|\mathrm{C}(V)| \text {. }
$$

Proof If $S$ and $T$ are subsets of $A$, then $v_{S} \cdot v_{T} \equiv|S \cap T|(\bmod 2)$. Thus for $S$ a non-empty subset of $V, S$ has the OIP $\Longleftrightarrow$ there exists $x \in V$ with $s \cdot x=1$ for all $s \in S$ $\Longleftrightarrow S \subseteq x^{\prime}$ for some $x \in V \backslash\{0\} \Longleftrightarrow S \subseteq B$ for some $B \in \mathcal{B}(V)$.

The identification $T \mapsto v_{T}$ depends on the basis $\left\{e_{i}\right\}$, as do the individual sets $x^{\prime}$. However the collection $\left\{x^{\prime} \mid x \neq 0\right\}=\mathcal{B}(V)$ is basis independent, as is equation (9). Thus for example $d(n-1)=|\mathcal{C}(W)|$ for any $W \in \mathcal{M}(V)$.

We now prove the recursion relation $(5)$ for $d(n)$.

Proof (Of (2)) For $k \geq 1$ define

$$
\mathcal{V}_{k}=\left\{S \in \mathcal{P}(V) \mid S \neq \emptyset \text { is a subset of exactly } 2^{k} V \text {-blocks }\right\} .
$$


By Lemma 2.3(c)

$$
\mathcal{V}_{k}=\left\{S \in \mathcal{P}(V) \mid S \text { is a } W \text {-subblock for exactly } 2^{k}-1 W \in \mathcal{M}(V)\right\} .
$$

We apply Lemma 2.1 twice, with $X=V$. First take $X=\mathcal{B}(V)$, and let $Q(S)$ be the property $S \neq \emptyset$. By Lemma 2.3 the set $z_{i}$ is empty except if $i=2^{k}$, and $z_{2^{k}}=\mathcal{V}_{k}$. Applying equation (9) gives

$$
d(n)=\left(2^{n}-1\right)\left(2^{2^{n-1}}-1\right)-\sum_{k \geq 1}\left(2^{k}-1\right)\left|\mathcal{V}_{k}\right| .
$$

Next take $\mathcal{X}=\mathcal{M}(V)$, and let $Q(S)$ hold if and only if $S \in \mathcal{C}(W)$ for some $W \in \mathcal{M}(V)$. By Lemma 2.2(c) if $Q(S)$ holds and $S \subseteq X_{j} \in \mathcal{X}$ then $S \in \mathfrak{C}\left(X_{j}\right)$, so $\mathcal{P}\left(X_{j}\right)^{Q}=\mathfrak{C}\left(X_{j}\right)$, and hence $\left|\mathcal{P}\left(X_{j}\right)^{Q}\right|=d(n-1)$. Furthermore by Lemma 2.3 the set $z_{i}$ is empty unless $i=2^{k}-1$ for some $k \geq 1$, and $Z_{2^{k}-1}=\mathcal{V}_{k}$. This gives

$$
\sum_{k \geq 1}\left(2^{k}-1\right)\left|\mathcal{V}_{k}\right|=\left(2^{n}-1\right) d(n-1) .
$$

Equation (5) follows from (12) and (13).

\section{The Even Intersection Property}

In this section we establish equation (3) for $e(n)$. Let $g(d)$ be the number of subsets of a $d$-dimensional $\mathbb{F}_{2}$-vector space $U$ that generate $U$. Under the identification $\mathcal{P}(A) \simeq V$,

$$
S \subseteq V \text { has the EIP } \Longleftrightarrow \emptyset \neq S \subseteq W \text { for some } W \in \mathcal{M}(V) .
$$

Thus a non-empty set $S \subseteq V$ does not have the EIP if and only if it generates $V$, so $g(n)=2^{2^{n}}-1-e(n)$ for $n \geq 1$. Since every subset $S \subseteq V$ gives rise to a subspace $U=\langle S\rangle$ in which (of course) $S$ generates $U$, summing over all generating subsets of all subspaces of $V$ gives

$$
\sum_{d=0}^{n}\left(\begin{array}{l}
n \\
d
\end{array}\right)_{2} g(d)=2^{2^{n}}
$$

We solve for $g(n)$. Taking $n=1,2, \ldots, m$ successively in equation (15) and subtracting the $d=0$ terms gives rise to the linear system

$$
B\left(\begin{array}{c}
g(1) \\
g(2) \\
\vdots \\
g(m)
\end{array}\right)=\left(\begin{array}{c}
2^{2^{1}}-2 \\
2^{2^{2}}-2 \\
\vdots \\
2^{2^{m}}-2
\end{array}\right),
$$

where $B$ is the $m \times m$ matrix in the next lemma. 
Lemma 3.1. Let $B$ be the lower triangular $m \times m$ matrix with $(i, j)$ entry $\left(\begin{array}{l}i \\ j\end{array}\right)_{2}$ if $i \geq j$, and remaining entries 0 . Then

$$
\left(B^{-1}\right)_{j k}= \begin{cases}(-1)^{j-k} 2^{\left(\begin{array}{c}
j-k \\
2
\end{array}\right)}\left(\begin{array}{l}
j \\
k
\end{array}\right)_{2}, & \text { if } j \geq k \\
0, & \text { otherwise. }\end{cases}
$$

Proof Let $C$ be the $m \times m$ matrix with entries given by the right hand side of (17). Clearly $B C$ is lower triangular, with 1's on the diagonal, so it remains to show $(B C)_{i k}=0$ for $i>k$. This follows from Cauchy's $q$-binomial Theorem [1, equation 10.0.9]:

$$
\sum_{h=0}^{N} q^{\left(\begin{array}{c}
h \\
2
\end{array}\right)}\left(\begin{array}{l}
N \\
h
\end{array}\right)_{q} t^{h}=\prod_{h=0}^{N-1}\left(1+q^{h} t\right)
$$

and the identity $\left(\begin{array}{l}i \\ j\end{array}\right)_{2}\left(\begin{array}{l}j \\ k\end{array}\right)_{2}=\left(\begin{array}{l}i \\ k\end{array}\right)_{2}\left(\begin{array}{l}i-k \\ j-k\end{array}\right)_{2}$. We have

$$
(B C)_{i k}=\sum_{j=k}^{i}\left(\begin{array}{l}
i \\
j
\end{array}\right)_{2}\left(\begin{array}{l}
j \\
k
\end{array}\right)_{2}(-1)^{j-k} 2^{\left(\begin{array}{c}
j-k \\
2
\end{array}\right)}=\left(\begin{array}{l}
i \\
k
\end{array}\right)_{2} \sum_{h=0}^{i-k} 2^{\left(\begin{array}{c}
h \\
2
\end{array}\right)}\left(\begin{array}{c}
i-k \\
h
\end{array}\right)_{2}(-1)^{h}=0
$$

where the last step follows from writing $h=j-k$ and applying (18) with $t=-1$ and $q=2$.

Applying Lemma 3.1 to equation (16) gives (3), and completes the proof in the even case.

\section{References}

[1] G. E. Andrews, R. Askey, R. Roy, Special Functions, Cambridge University Press, 2000.

[2] C. Colbourn, J. Dinitz (Eds.), The CRC Handbook of Combinatorial Designs, CRC Press, 1996.

[3] S. Elaydi, An Introduction to Difference Equations, third ed., Springer, 2005.

[4] J. Goldman, G-C. Rota, The number of subspaces of a vector space, Recent Progress in Combinatorics, Ed. W. T. Tutte, Academic Press (1969) 75-83.

[5] R. Graham, M. Grötschel, L. Lovász (Eds.), Handbook of Combinatorics, Vol. 1, MIT Press, 1995.

[6] S. Hu, A note on the $d$ th power residue symbol of $\mathbb{F}_{q}[t]$, J. Number Theory 128 (2008) 2655-2662.

[7] R. Mickens, Difference Equations, Theory and Applications, second ed., Chapman \& Hall, 1991.

[8] S. Roman, Field Theory, second ed., Springer, 2006. 
[9] S. Wright, Patterns of quadratic residues and nonresidues for infinitely many primes, J. Number Theory 123 (2007) 120-132.

[10] S. Wright, Quadratic residues and the combinatorics of sign multiplication, J. Number Theory 128 (2008) 918-925.

[11] S. Wright, Some Enumerative Combinatorics Arising From a Problem on Quadratic Nonresidues, Australasian J. Combinatorics, 44 (2009) 301-315. 